\title{
Compositional Changes for Reduction of Polymerisation-Induced Shrinkage in Holographic Photopolymers
}

\author{
D. Cody, ${ }^{1,2}$ M. Moothanchery, ${ }^{1}$ E. Mihaylova, ${ }^{1,3}$ V. Toal, ${ }^{1,2}$ S. Mintova, ${ }^{4}$ and I. Naydenova ${ }^{1,2}$ \\ ${ }^{1}$ Centre for Industrial and Engineering Optics, Dublin Institute of Technology, Dublin 8, Ireland \\ ${ }^{2}$ School of Physics, Faculty of Science, Dublin Institute of Technology, Dublin 8, Ireland \\ ${ }^{3}$ Department of Mathematics, Informatics and Physics, Agricultural University, Plovdiv, Bulgaria \\ ${ }^{4}$ LCS, CRISMAT, University of Caen, 6 boulevard du Maréchal Juin, 14050 Caen Cedex, France
}

Correspondence should be addressed to I. Naydenova; izabela.naydenova@dit.ie

Received 24 March 2016; Revised 30 July 2016; Accepted 17 August 2016

Academic Editor: Sergi Gallego

Copyright (c) 2016 D. Cody et al. This is an open access article distributed under the Creative Commons Attribution License, which permits unrestricted use, distribution, and reproduction in any medium, provided the original work is properly cited.

\begin{abstract}
Polymerisation-induced shrinkage is one of the main reasons why many photopolymer materials are not used for certain applications including holographic optical elements and holographic data storage. Here, two compositional changes for the reduction of shrinkage in an acrylamide-based photopolymer are reported. A holographic interferometric technique was used to study changes in the dynamics of the shrinkage processes occurring in the modified photopolymer during holographic recording in real time. Firstly, the effect of the replacement of the acrylamide monomer in the photopolymer composition with a larger monomer molecule, diacetone acrylamide, on polymerisation-induced shrinkage has been studied. A reduction in relative shrinkage of 10$15 \%$ is obtained using this compositional change. The second method tested for shrinkage reduction involved the incorporation of BEA-type zeolite nanoparticles in the acrylamide-based photopolymer. A reduction in relative shrinkage of $13 \%$ was observed for acrylamide photopolymer layers doped with $2.5 \%$ wt. BEA zeolites in comparison to the undoped photopolymer.
\end{abstract}

\section{Introduction}

Holography has become an area of great interest with application in many fields including holographic digital microscopy $[1,2]$, holographic tweezers [3], holographic optical elements (HOEs) for solar concentrators $[4,5]$, sensors [6-10], and data storage (HDS) [11, 12]. A large range of photosensitive media are available for holographic recording, each with its inherent advantages and disadvantages. Of these, photopolymer materials have been the subject of much study for holographic applications due to their large dynamic range, high sensitivity, and self-processing nature. Acrylamide(AA-) based photopolymers in particular have been very popular among the research community due to the fact that they are water-soluble and easy to prepare and have relatively low cost and readily record bright, efficient holograms.

A drawback of photopolymer media is that they suffer from polymerisation-induced shrinkage [13-17]. As polymer chains form in the illuminated regions of a holographic grating during exposure, the density of the region is increased. The individual monomer molecules polymerise in a chain formation and become tightly packed together, resulting in shrinkage of the photopolymer layer. This shrinkage alters the diffraction properties of the grating as it is recorded. Shrinkage in photopolymer media restricts their suitability for certain holographic applications. If the recording medium suffers from shrinkage during recording, then the exact angle of recording and therefore the angle of reconstruction are shifted, and so read-out of the holograms is not possible without additional compensation for this shift. For example, shrinkage of HOEs during the fabrication process results in dimensional changes in the holographic element. This is a problem also for HDS, a technique which utilises the fact that holograms have high angular selectivity. Currently the upper limit for shrinkage in commercial HDS materials is $0.5 \%$ [18]. 
Here, two methods for reduction of shrinkage in a watersoluble photopolymer system are reported. A holographic interferometric technique developed by Moothanchery et al. is used to quantify the relative shrinkage occurring in photopolymer compositions during holographic recording in real time [19].

The first method for shrinkage reduction involves replacement of the AA main monomer in the photopolymer composition with a larger monomer molecule, diacetone acrylamide (DA). Previous studies reported by the authors have shown that substitution with the DA monomer molecule impacts on grating formation during holographic recording, reducing both the rate of polymerisation and the rate of diffusion [20-22]. This is attributed to the larger size of the DA molecule $(10 \times 4 \AA)$, which is approximately double the length of the AA monomer $(5 \times 4 \AA)$. It is expected that the replacement of the AA monomer with DA will influence the amount of polymerisation-induced shrinkage which occurs as a result of holographic recording due to variation of the monomer size and, therefore, the size and density of the resultant polymer chains. Comparison is made with previous measurements of shrinkage in an AA-based photopolymer system [23].

The second method for shrinkage reduction involves the incorporation of BEA-type zeolite nanoparticles in the AAbased photopolymer. The idea of incorporating nanoparticles in photopolymer media was first introduced in 1994 by Krug and Schmidt [24] and in 1996 by Oliveira et al. [25]. In their photopolymer system, monomer molecules containing $\mathrm{ZrO}_{2}$ nanoparticles were found to diffuse from the dark to the bright fringe regions during holographic recording, since the methacrylic component at the surface of the $\mathrm{ZrO}_{2}$ nanoparticles being formed by the in situ condensation of methacrylic acid complexed zirconium propoxide was used to copolymerise with the organic species of the organosilane. On the other hand, Suzuki et al. [26-28] and later Sánchez et al. [29] among others examined the dispersion of inorganic nanoparticles to increase the refractive index modulation, $\Delta n$, by the counter diffusion of monomer and nanoparticles during holographic recording (i.e., monomer diffusion from the dark to the bright fringe regions and nanoparticle diffusion from the bright to the dark fringe regions). A detailed review of photopolymerisable nanocomposite photonic materials can be found in [30]. Suzuki et al. demonstrated that the inclusion of $\mathrm{TiO}_{2}, \mathrm{SiO}_{2}$, and $\mathrm{ZrO}_{2}$ nanoparticles in their (meth)acrylate photopolymer led to a reduction in the amount of polymerisation-induced shrinkage, which was measured using the Bragg curve shift method [26-28]. Other methods for the reduction of shrinkage in holographic photopolymers include the use of epoxy resins [31] and organic-inorganic hybrid matrices [16]. Hata and Tomita demonstrated that substantial shrinkage suppression of nanoparticle-doped thiol-ene photopolymer systems can be achieved by use of step-growth polymerisation mechanisms in combination with the dispersion of nanoparticles [32]. Moothanchery et al. previously reported significant reduction in the shrinkage of an AA photopolymer with the incorporation of silica MFI-type zeolites (silicalite-1). Using the Bragg curve shift method, shrinkage of the zeolite-doped AA

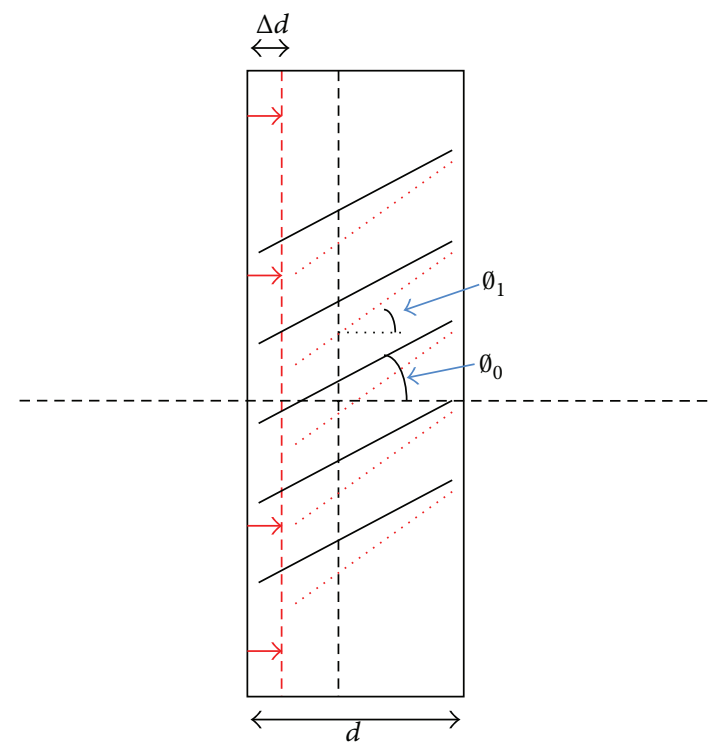

FIGURE 1: Schematic diagram showing reorientation of fringes in a transmission grating as a result of shrinkage. $\emptyset_{0}$ and $\emptyset_{1}$ are the initial and final slant angles.

photopolymer has been shown to decrease from 1.32 to $0.57 \%$, just above the commercial limit on shrinkage for HDS [33].

Here, for the first time, a highly accurate holographic interferometric technique has been used to characterise in real time the effect of BEA-type zeolite nanoparticles and a larger DA monomer molecule on the shrinkage occurring in photopolymer films during holographic recording. In comparison to the Bragg method, this real-time technique allows for studying the dynamics of the shrinkage processes. Comparison is made regarding the relative effectiveness of the two methods for suppressing polymerisation-induced shrinkage.

\section{Theory}

2.1. Quantification of Shrinkage in Photopolymer Systems. Extensive experimental work has been carried out to develop methods with which to accurately quantify the amount of shrinkage occurring in different photopolymer systems. Zhao et al. calculated shrinkage in the DuPont photopolymer to be $5.25 \%$ using the Bragg curve shift method [34]. In this widely used method, the fractional change $\Delta d$ in the sample thickness $d$ is calculated from monitoring the shift between the initial $\emptyset_{0}$ and final $\emptyset_{1}$ slant angles:

$$
\frac{\Delta d}{d}=\frac{\tan \emptyset_{1}}{\tan \emptyset_{0}}-1
$$

$\emptyset_{0}$ and $\emptyset_{1}$, as shown in Figure 1, are determined from the position of the Bragg curve peak before and after shrinkage occurs due to exposure [17]. The method assumes a dimensional change in the layer in the direction normal to the layer's surface. The change in the plane of the layer is usually ignored, although Shelby et al. have shown that inplane shrinkage also occurs [35]. This method was previously used by the authors to measure the relative shrinkage of an 
AA-based photopolymer, producing a value of $1.32 \%$ [19], which is significantly lower than that of the DuPont photopolymer.

Gallego et al. have developed a novel method of measuring shrinkage in an acrylate formulation using a reflection interferometer setup [36]. Shrinkage values of up to $3 \%$ were measured, and it was observed that volume shrinkage decreases with increasing spatial frequency of recording. A spectral interferometric method which uses a white-light Fabry-Pérot interferometer for measurement of shrinkage evolution in a photopolymer system is reported by Kawana et al. [37]. They report discrepancies between the spectral interferometric and holographic Bragg-angle detuning measurements of the polymerisation-induced shrinkage.

\subsection{Holographic Interferometry for Real-Time Measurement} of Shrinkage. Holographic interferometry is a noncontact technique which can be used to study surfaces which are not optically smooth with very high precision and sensitivity. Its discovery is accredited to Leith and Upatnieks in 1963 [38], but it was not until the work of Powell and Stetson in 1965 that the observed phenomenon was explained [39]. Several theoretical models have been developed for fringe analysis and evaluation of experimental holographic interferometry: the fringe plane rotation model [40], the fringe plane rotation and average refractive index change model [41, 42], and the fringe bending model [43].

A holographic interferometric method has been developed by Moothanchery et al. [23, 44], which allows monitoring of the shrinkage of the photopolymer layer in real time. In this method, a hologram of the object is recorded at a wavelength of $633 \mathrm{~nm}$; in this case, the object is a photopolymer layer with negligible absorbance at this wavelength. A simple grating is then recorded in the object itself at a wavelength of $532 \mathrm{~nm}$. This causes the photopolymer layer (i.e., the object) to shrink, changing the optical path length $L$ of the $633 \mathrm{~nm}$ object beam. This object beam, which is changing in real time, is caused to interfere with the beam reconstructed from the hologram of the unperturbed object. This produces interference fringes on the reconstructed image from the hologram, which are captured in real time using a CMOS camera. Figure 2 shows an example of images from a hologram of an AAbased photopolymer layer surface before exposure and again after exposure, in which the interference fringes are visible. From these captured interferograms, it is possible to calculate the change in the optical path length $L$ of the object beam from the number of fringes $n$ via the following equation:

$$
L=n \lambda,
$$

where $\lambda$ is the wavelength of the laser used in this experiment. Equation (3) relates the optical path length to the absolute shrinkage in microns $\Delta d$ of the photopolymer layer:

$$
L=\Delta d\left(\cos \theta_{1}+\cos \theta_{2}\right),
$$

where $\theta_{1}$ is the angle of illumination and $\theta_{2}$ is the angle of reconstruction (Figure 3). By combining (2) and (3), the absolute shrinkage can be directly calculated from the number of fringes $n$ :

$$
\begin{aligned}
& n \lambda=\Delta d\left(\cos \theta_{1}+\cos \theta_{2}\right), \\
& \Delta d=\frac{n \lambda}{\left(\cos \theta_{1}+\cos \theta_{2}\right)} .
\end{aligned}
$$

The absolute shrinkage $\Delta d$ of the photopolymer layer is thus determined from the number of fringes $n$ observed in the captured images for specified values of $\lambda, \theta_{1}$, and $\theta_{2}$ in (5). This allows for the shrinkage as a function of time to be studied by noting the time at which the interferometric fringes $(n=1,2, \ldots)$ appear in the live video, as seen in Figure 2(c). This real-time technique is advantageous over the Bragg curve shift method for measuring shrinkage as it allows for dimensional changes in the material to be monitored as they occur and therefore provides more information about the dynamics of the shrinkage processes rather than just a single measurement made after recording has finished.

\section{Experimental}

\subsection{Materials}

3.1.1. Material Preparation for Composition A: Monomer Substitution. It was necessary to prepare two different types of photopolymer samples for this experiment, as two different laser wavelengths are used. A green light-sensitised DA photopolymer solution was prepared for recording at $532 \mathrm{~nm}$, as shrinkage is measured in this layer. For the $633 \mathrm{~nm}$ recordings which produce the interferograms of the DA photopolymer layers, an AA-based photopolymer solution was used. This was done to allow for accurate comparison with shrinkage measurements for the AA photopolymer by using the same recording conditions and recording material in which to record a hologram of the unexposed DA photopolymer surface. The two photopolymer compositions used are described in Table 1. The DA photopolymer layers were prepared by depositing $0.5,0.8$, and $1.4 \mathrm{~mL}$ of solution onto $75 \times 25 \mathrm{~mm}$ glass microscope slides to produce dry sample thicknesses of 45,90 , and $120 \pm 5 \mu \mathrm{m}$. The AA photopolymer layers were prepared by depositing $0.6 \mathrm{~mL}$ of photopolymer solution onto the same glass slides. The thickness of the dry photopolymer layer was measured using a white-light surface profiler (Micro XAM S/N 8038) and was found to be $60 \pm$ $5 \mu \mathrm{m}$. The layers were dried for approximately 24 hours before recording at room temperature.

3.1.2. Material Preparation for Composition B: Incorporation of Nanoparticles. A green light-sensitised AA-based photopolymer was prepared by addition of $0.6 \mathrm{~g}$ of AA monomer, $2 \mathrm{~mL}$ of triethanolamine, $0.2 \mathrm{~g}$ of $\mathrm{N}, \mathrm{N}$-methylenebisacrylamide, and $4 \mathrm{~mL}$ of erythrosin B dye $(0.11 \%$ wt. water stock solution) to $9 \mathrm{~mL}$ stock solution of polyvinyl alcohol in water (20\% wt.). The synthesis of the BEA-type zeolite nanoparticles is described in [45]. The zeolite nanoparticles were added to $1 \mathrm{~mL}$ volumes of the green light-sensitised AA photopolymer solution as colloidal aqueous suspensions in volumes of $0.067,0.169,0.347$, and $0.531 \mathrm{~mL}$ containing 


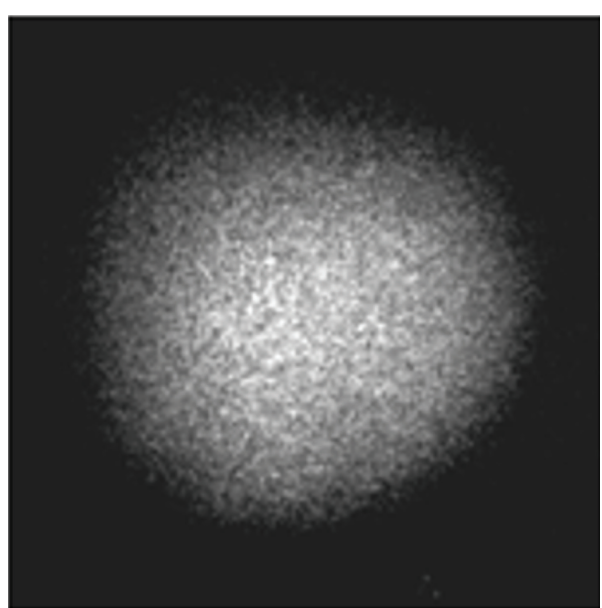

(a)

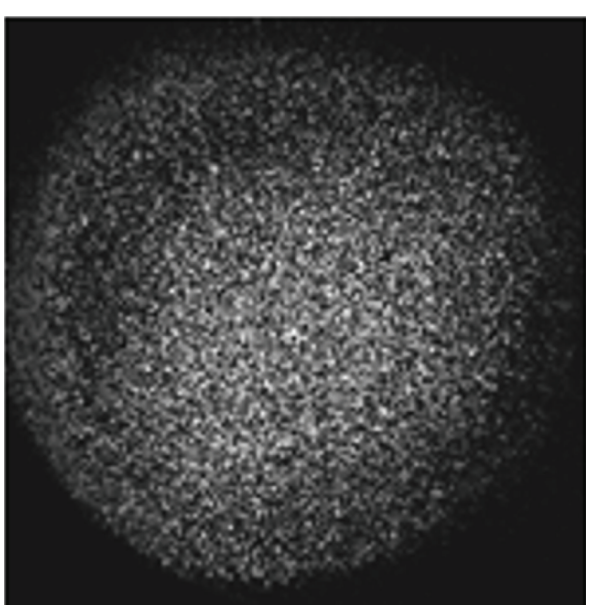

(b)

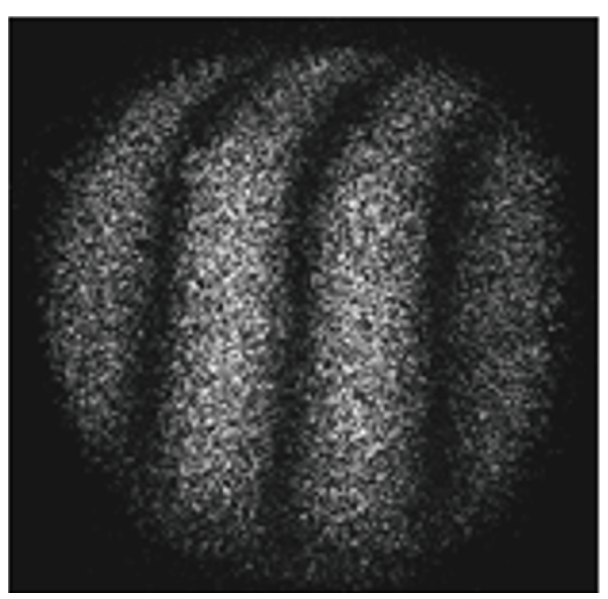

(c)

FIGURE 2: (a) Reference frame, produced by capturing the beam reconstructed from the hologram recorded in the red sensitive layer. (b) Result of subtraction of the reference frame from the current frame (before switching on the green laser). (c) Fringes visible as a result of subtraction of the reference frame from the current frame (after switching on the green laser).

TABLE 1: DA and AA photopolymer solutions for shrinkage measurements.

\begin{tabular}{lcc}
\hline (All in moles/L) & $532 \mathrm{~nm}$-sensitised DA photopolymer & 633 nm-sensitised AA photopolymer \\
\hline 10\% wt./vol polyvinyl alcohol & 1.73 & 1.53 \\
Triethanolamine & 0.58 & 0.58 \\
Diacetone acrylamide & 0.23 & - \\
Acrylamide & - & 0.32 \\
$N, N^{\prime}$-Methylenebisacrylamide & 0.05 & 0.05 \\
$0.11 \%$ wt./vol erythrosin B dye s.s. & $2.02 \times 10^{-4}$ & - \\
$0.11 \%$ wt./vol methylene blue dye s.s. & - & $5.29 \times 10^{-4}$ \\
\hline
\end{tabular}

$4.9 \%$ wt. of nanoparticles. Additional water was added to equalize the solid substance concentrations in all stock solutions and to obtain the same thickness for the final nanocomposite layers. For sample preparation, $3.5 \mathrm{~mL}$ of BEA nanocomposite solution was spread on $25 \times 120 \mathrm{~mm}$ glass plates and allowed to dry for 24 hours. The thickness of the dried BEA nanocomposite layers was $160 \pm 5 \mu \mathrm{m}$. The final concentrations of zeolite nanoparticles were $0,1,2.5,5$, and
7.5\% wt. Samples of a red light-sensitised AA were prepared as described in Section 3.1.1.

3.2. Methods. The holographic interferometer setup used is shown in Figure 4. The 532 and $633 \mathrm{~nm}$ beams were spatially filtered, collimated, and then split in two using beam splitters. The two $633 \mathrm{~nm}$ beams were used in an interferometric arrangement to monitor the changes due to shrinkage in the 


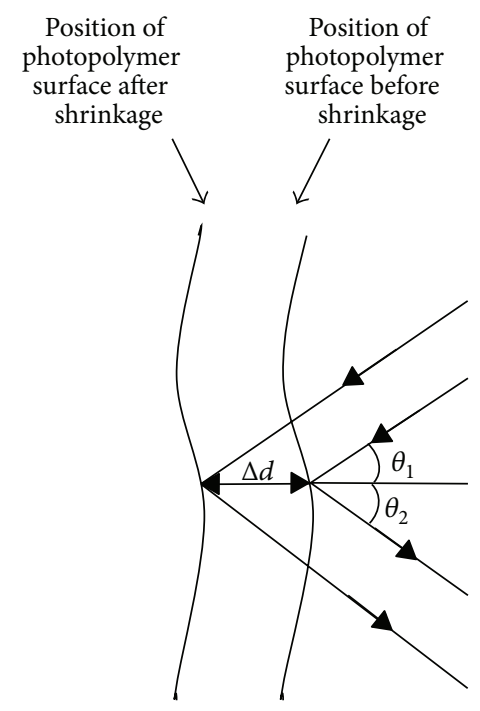

FIGURE 3: Illustration of change in optical path due to shrinkage.

green light-sensitised sample during exposure. To do this, one of the $633 \mathrm{~nm}$ beams was reflected from the surface of the green light-sensitised sample (object beam). The other $633 \mathrm{~nm}$ beam (reference beam) was partially reflected from a glass plate so as to allow for adjustment of the intensity and directed so that it interfered with the object beam of matching intensity on the red light-sensitised sample, thus recording a hologram of the surface of the green light-sensitised sample. An exposure time of 140 seconds and intensity of $0.5 \mathrm{~mW} / \mathrm{cm}^{2}$ were used for this recording. It is important to note that the green light-sensitised layer is not affected by the red beam, since the absorption at this wavelength is negligible. Using the $532 \mathrm{~nm}$ interferometer, a grating was then recorded in the green light-sensitised sample, causing it to shrink. The real-time $633 \mathrm{~nm}$ object beam from the shrinking green light-sensitised layer was then allowed to interfere with the reconstructed object beam from the hologram of the unexposed green light-sensitised photopolymer layer on a screen. This allowed the resulting interferograms to be videorecorded in real time using a CMOS camera. The angles of illumination and observation of the $633 \mathrm{~nm}$ object beam were $26.2^{\circ}$ and $33.7^{\circ}$, respectively. An image subtraction method was used in order to overcome the limited visibility of the fringes. A reference frame of the hologram recorded in the red light-sensitised sample (before the $532 \mathrm{~nm}$ exposure began) was subtracted using LabVIEW software from the other live frames to achieve this. A comprehensive description of the LabVIEW program used can be found elsewhere [23]. The intensity of the $532 \mathrm{~nm}$ laser was controlled using a neutral density filter.

Equation (6) was used to calculate the diffraction efficiency, $\eta$, of the transmission gratings recorded at $532 \mathrm{~nm}$ :

$$
\eta=\frac{I_{d}}{I_{o}} \times 100
$$

where $I_{o}$ is the incident intensity of a $633 \mathrm{~nm}$ probe beam and $I_{d}$ is the intensity of light diffraction by the grating.

\section{Results and Discussion}

4.1. Results for Composition A: Effect of Monomer Substitution on Shrinkage. Measurement of the polymerisation-induced shrinkage of the DA photopolymer was carried out for different values of layer thickness and recording intensity. Two different definitions of shrinkage are used to characterise the samples: relative shrinkage, measured as a percentage of the overall sample thickness, and absolute shrinkage, the actual change in sample thickness measured in microns. Due to variations in dry sample thickness of $\pm 5 \mu \mathrm{m}$, the absolute and relative shrinkage values have an associated error of up to $\pm 0.1 \%$.

Figure 5 shows the influence of sample thickness on both relative shrinkage and absolute shrinkage, which occurs during holographic recording in real time for three sample thicknesses: 45,90 , and $120 \mu \mathrm{m}$. Both the relative shrinkage and absolute shrinkage are observed to increase with increasing sample thickness for gratings recorded in the DA layers with a recording intensity of $1 \mathrm{~mW} / \mathrm{cm}^{2}$. Low recording intensities such as this have previously been shown to maximise polymerisation-induced shrinkage [44] and so were purposely chosen here in order to facilitate study of the shrinkage dynamics.

The same trend of increasing relative shrinkage with sample thickness was also observed for higher recording intensities of 5 and $10 \mathrm{~mW} / \mathrm{cm}^{2}$ (Figure 6). This is the opposite of the trend observed by Moothanchery et al. previously for the AA photopolymer [23]. This trend is most likely due to the fact that the light intensity and thus the number of photons penetrating the sample decrease exponentially with sample thickness. Figure 7 shows the relative shrinkage versus exposure energy for gratings recorded with intensities of 1 , 5 , and $10 \mathrm{~mW} / \mathrm{cm}^{2}$ in $120 \mu \mathrm{m}$ thick DA photopolymer layers. The amount of relative shrinkage at $1 \mathrm{~mW} / \mathrm{cm}^{2}$ is significantly greater than that at higher recording intensities for the same exposure energy. An identical trend was observed for 45 and $90 \mu \mathrm{m}$ thick layers. At lower intensities, the concentration of free radicals is reduced, and so longer polymer chain growth is promoted. This is due to the fact that there is a lower volume of free radicals resulting in a lower rate of termination. Thus, for the same exposure energy absorbed by the layer, the relative shrinkage at lower intensity is effectively larger than that caused by illumination with higher intensity. This intensity dependence is the same as the trend observed for the AA photopolymer by Moothanchery et al. [23]. On the basis of this dependence of relative shrinkage on intensity, one can analyse the results for layers of different thicknesses as shown in Figures 5 and 6. Increased relative shrinkage is observed for thicker samples as a result of the effective lower recording intensity in the furthermost sections of the layer.

For recording intensities of 1 and $5 \mathrm{~mW} / \mathrm{cm}^{2}$, the increase in relative shrinkage with thickness is equidistant. However, in the case of the $10 \mathrm{~mW} / \mathrm{cm}^{2}$ data, the same approximate maximum shrinkage value is reached for both the 45 and $90 \mu \mathrm{m}$ thickness layers. This result has been observed in several samples and is repeatable. One possible explanation is that as the recording intensity is increased to $10 \mathrm{~mW} / \mathrm{cm}^{2}$, 


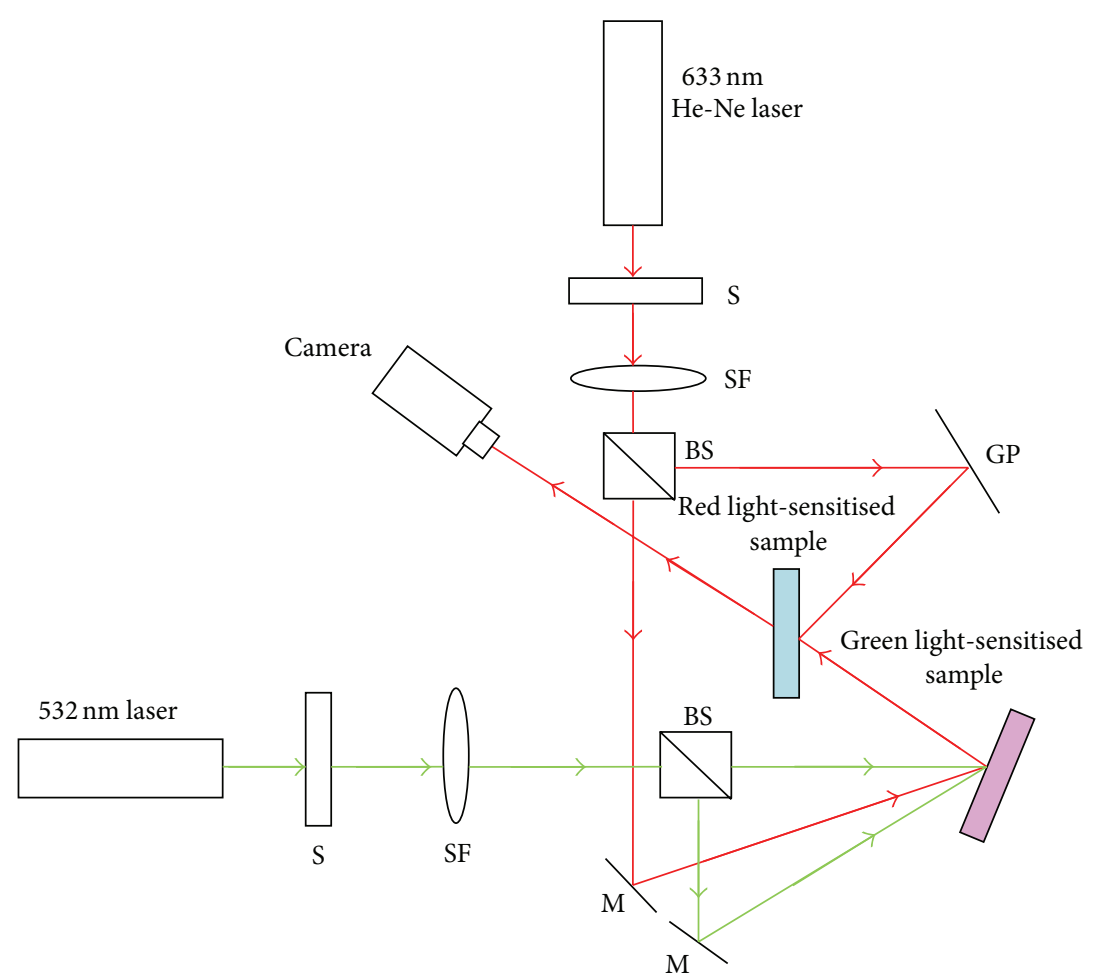

FIGURE 4: Holographic interferometry setup; S, shutter, SF, spatial filter, BS, beam splitter, GP, glass plate, and M, mirror.
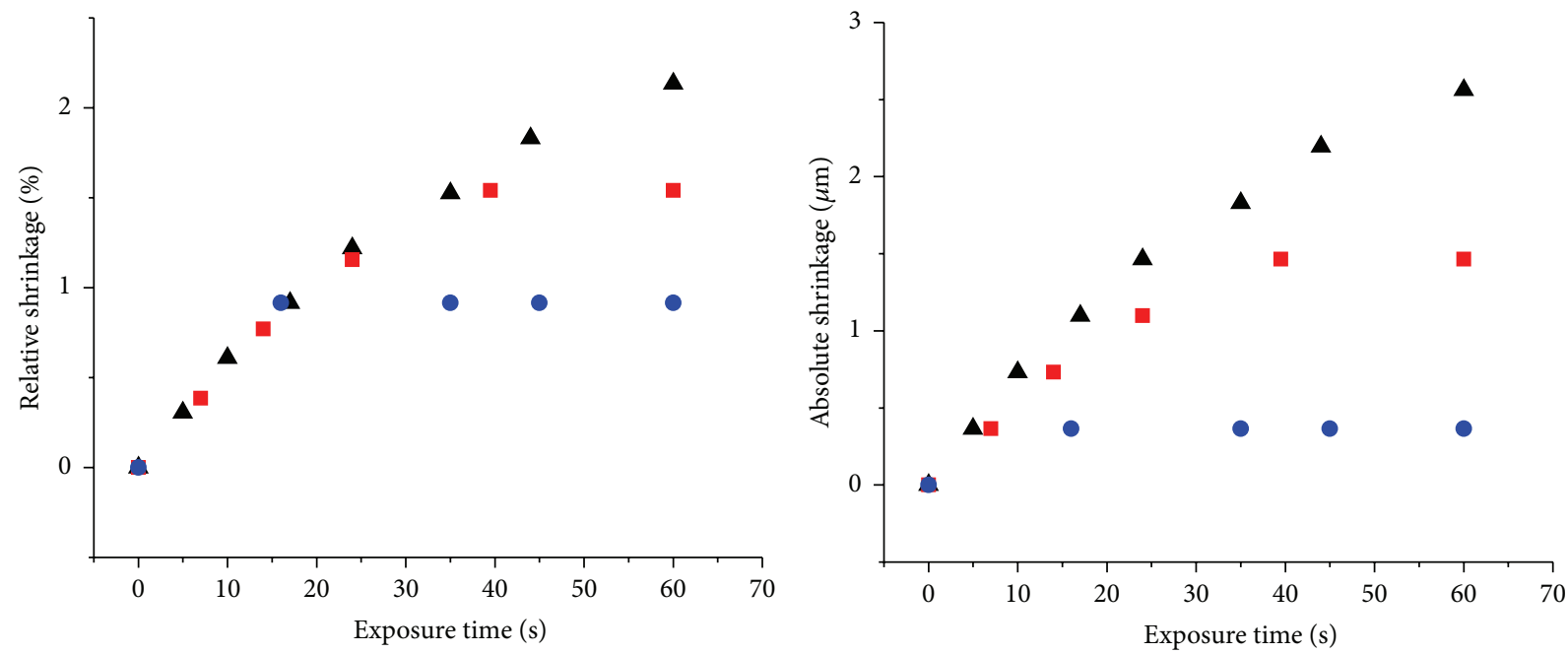
- $120 \mu \mathrm{m}$
- $90 \mu \mathrm{m}$
- $45 \mu \mathrm{m}$
- $120 \mu \mathrm{m}$
$90 \mu \mathrm{m}$
- $45 \mu \mathrm{m}$

(a)

(b)

FIGURE 5: (a) Relative shrinkage (\%) versus exposure time (s) and (b) absolute shrinkage ( $\mu \mathrm{m})$ versus exposure time (s) for gratings recorded in DA photopolymer layers with thickness of 45,90 , and $120 \mu \mathrm{m}$ using a recording intensity of $1 \mathrm{~mW} / \mathrm{cm}^{2}$.

more photons of light will penetrate the furthermost sections of the layer. Therefore, the dependence of relative shrinkage on thickness will become less pronounced with increasing intensity.
From Figure 7, it is also seen that there is a much larger difference observed in the rate of relative shrinkage between $1 \mathrm{~mW} / \mathrm{cm}^{2}$ and $5 \mathrm{~mW} / \mathrm{cm}^{2}$ than between $5 \mathrm{~mW} / \mathrm{cm}^{2}$ and $10 \mathrm{~mW} / \mathrm{cm}^{2}$. This indicates that there exists an optimum 


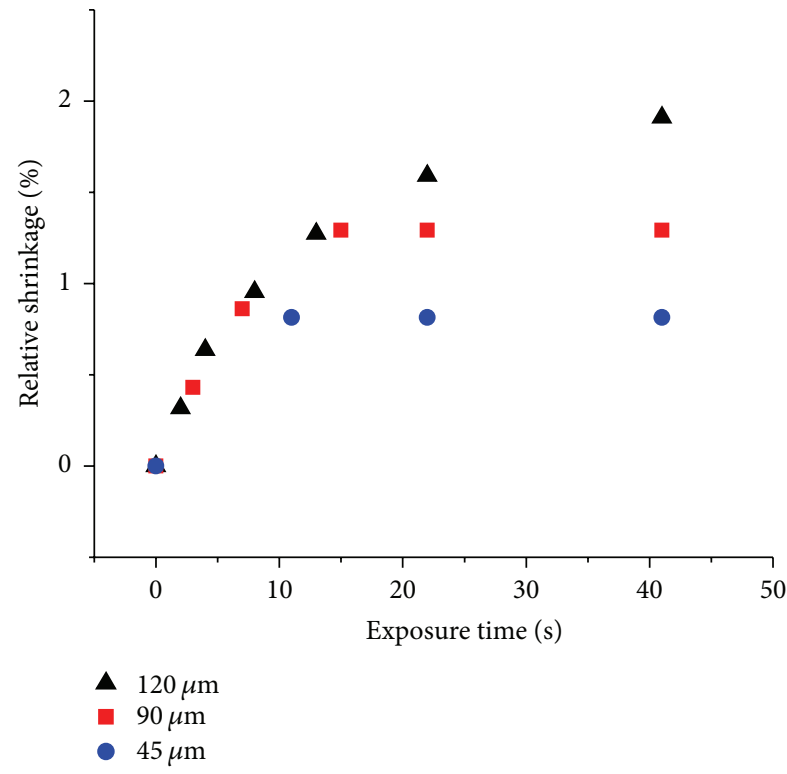

(a)

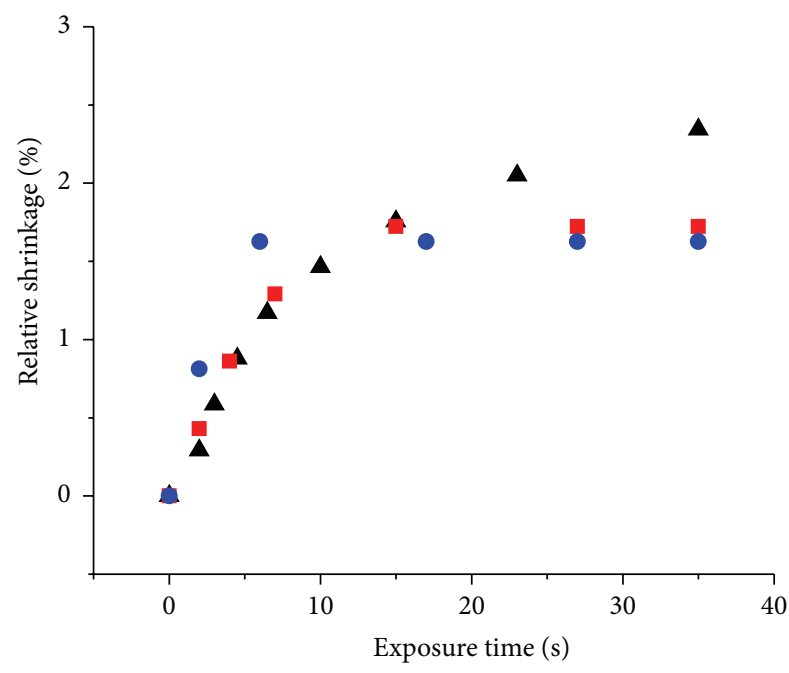
$\Delta 120 \mu \mathrm{m}$
- $90 \mu \mathrm{m}$
- $45 \mu \mathrm{m}$

FIGURE 6: Relative shrinkage (\%) versus exposure time (s) for gratings recorded in 45, 90, and $120 \mu \mathrm{m}$ DA photopolymer layers using a recording intensity of (a) $5 \mathrm{~mW} / \mathrm{cm}^{2}$ and (b) $10 \mathrm{~mW} / \mathrm{cm}^{2}$.

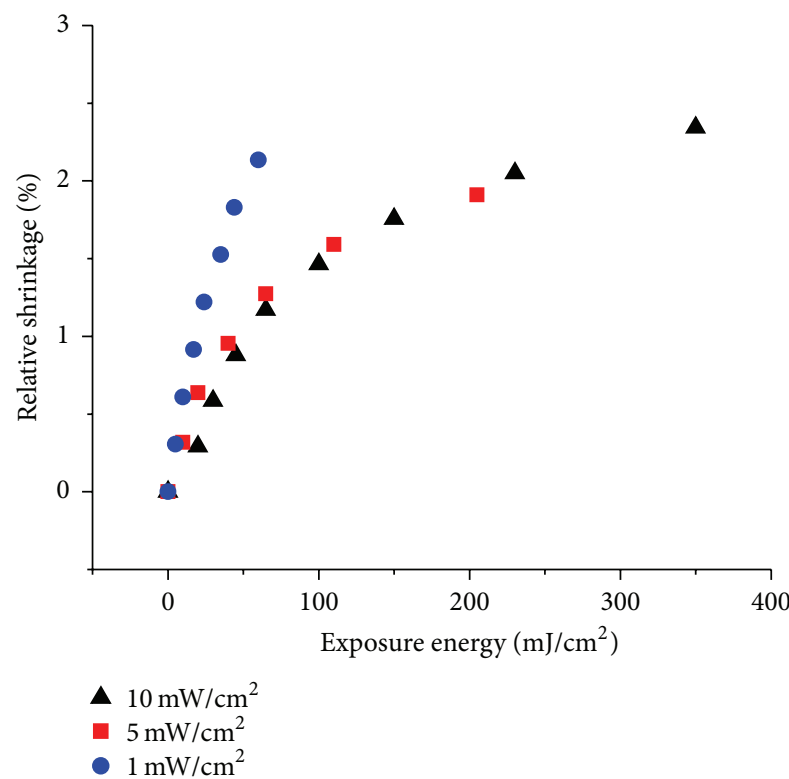

FIGURE 7: Relative shrinkage (\%) versus exposure energy $\left(\mathrm{mJ} / \mathrm{cm}^{2}\right)$ for gratings recorded in $120 \mu \mathrm{m}$ DA photopolymer layers with recording intensities of 1,5 , and $10 \mathrm{~mW} / \mathrm{cm}^{2}$.

recording intensity beyond which further increase of the intensity will have a limited effect on the rate of relative shrinkage as a function of exposure energy.

In order to investigate the different processes occurring in the DA photopolymer films during holographic recording and to allow for comparison with the AA photopolymer, the relative shrinkage versus exposure time data for the DA photopolymer was fitted with a double exponential function of the following form:

$$
y=y_{o}-A_{1} e^{\left(-x / t_{1}\right)}-A_{2} e^{\left(-x / t_{2}\right)},
$$

where $y_{o}$ is the final relative shrinkage value, $A_{1}$ and $A_{2}$ are the amplitudes of the first and second processes, and $t_{1}$ and $t_{2}$ are the time constants for the first and second processes. Initially a single exponent function was fitted; however, this was observed to be a poor fit. This is most likely because there are two processes taking place which determine the dynamics of the photopolymer shrinkage, especially for higher recording intensities. The first process is characterised by a time constant of a few seconds $\left(t_{1}\right)$, while the second process is characterised by a time constant in the order of tens of seconds $\left(t_{2}\right)$. Moothanchery et al. ascribed the slower second process, characterised by the longer time constant, to the conversion of monomer to polymer. They observed that as the recording intensity is increased, the value for the second time constant decreases as expected with faster monomer to polymer conversion. The first, faster process which is observed at higher recording intensities is thought to be governed by the rate of crosslinking of the AA photopolymer composition [23].

Here, a double exponential function has been fitted to the relative shrinkage versus exposure time data for the $120 \mu \mathrm{m}$ thick DA photopolymer layers to allow for comparison with data for the $110 \mu \mathrm{m}$ thick AA photopolymer layers for recording intensities of 1,5 , and $10 \mathrm{~mW} / \mathrm{cm}^{2}$. The fitted curves are shown in Figure 8 and the extracted values of the five parameters are given in Table 2.

From the results for the DA photopolymer, it is seen that as was the case for AA the process characterised by the shorter 
TABLE 2: Data from double exponential fit of relative shrinkage versus exposure time.

\begin{tabular}{ccccccc}
\hline & Intensity $\left(\mathrm{mW} / \mathrm{cm}^{2}\right)$ & $y_{o}(\%)$ & $A_{1}$ & $t_{1}(\mathrm{~s})$ & $A_{2}$ & $t_{2}(\mathrm{~s})$ \\
\hline \multirow{3}{*}{ DA } & 1 & $2.9 \pm 0.2$ & NA & NA & $2.9 \pm 0.1$ & $45 \pm 5$ \\
& 5 & $2.2 \pm 0.8$ & $0.8 \pm 1.4$ & $4.9 \pm 8$ & $1.5 \pm 0.8$ & $25 \pm 49$ \\
& 10 & $2.88 \pm 0.09$ & $1.40 \pm 0.07$ & $2.8 \pm 0.3$ & $1.94 \pm 0.04$ & $22 \pm 3$ \\
\hline \multirow{4}{*}{ AA } & 1 & $3.30 \pm 0.03$ & NA & NA & $3.40 \pm 0.03$ & $62.50 \pm 0.01$ \\
& 5 & $3.30 \pm 0.05$ & $0.86 \pm 0.08$ & $9.60 \pm 0.01$ & $2.44 \pm 0.05$ & $71.40 \pm 0.01$ \\
& 10 & $2.8 \pm 0.1$ & $1.3 \pm 0.3$ & $3.0 \pm 0.2$ & $1.8 \pm 0.2$ & $34.40 \pm 0.01$ \\
\hline
\end{tabular}

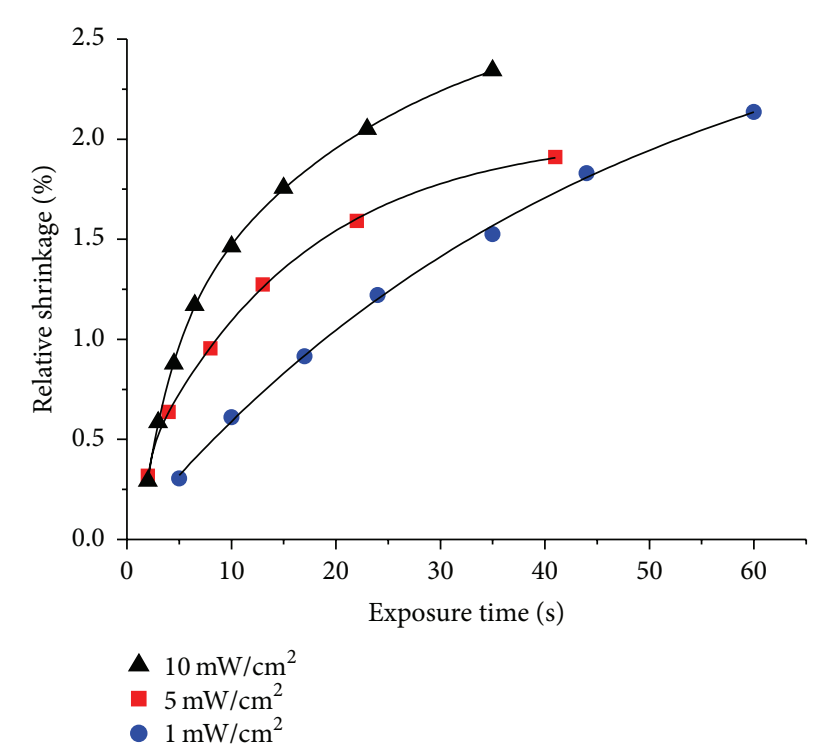

FIGURE 8: Relative shrinkage (\%) versus exposure time (s) for gratings recorded in $120 \mu \mathrm{m}$ DA photopolymer layers with recording intensities of 1,5 , and $10 \mathrm{~mW} / \mathrm{cm}^{2}$, fitted with a double exponential function.

time constant was not evident at the lowest recording intensity tested of $1 \mathrm{~mW} / \mathrm{cm}^{2}$. For such low recording intensities, the rate at which the free radicals are generated will be lower and hence the crosslinking process will be slower. For this reason, a single exponential fit is sufficient to fit the dynamics of the shrinkage process at such low intensity of recording.

As the recording intensity is increased to $5 \mathrm{~mW} / \mathrm{cm}^{2}$, two processes appear to be in operation. As the recording intensity is increased, the rate of free radical generation is faster and the density of free radicals per unit volume will be higher, resulting in faster and greater extent of crosslinking of the polymer chains. This is reflected in the faster process observed in the shrinkage dynamics. However, it is not possible to draw any more conclusions regarding a trend in the crosslinking rate due to the large error on both the time constant and amplitude values for the $5 \mathrm{~mW} / \mathrm{cm}^{2}$ data. This large error is due to the fact that there are very few data points available for a double exponential fit. As the recording intensity is increased again to $10 \mathrm{~mW} / \mathrm{cm}^{2}$, the faster crosslinking process becomes more prevalent; however, its significance is still small relative to the second process, which is associated with the rate of DA monomer to polymer conversion.
By comparing the final relative shrinkage values for the DA and AA photopolymers at all intensities, it is observed that the DA photopolymer on average demonstrates approximately $10-15 \%$ less relative shrinkage as a percentage of sample thickness than the AA photopolymer. This is most likely attributed to the ability of the smaller AA monomer molecules to diffuse at a faster rate, thereby promoting the growth of longer polymer chains, resulting in increased relative shrinkage in comparison to the DA photopolymer.

Additionally, the relationship between grating diffraction efficiency, $\eta$, and relative shrinkage was investigated for the AA and DA photopolymers. Table 3 shows the relative shrinkage measured for increasing values of grating $\eta$. The gratings were recorded with exposure energies of $25-200 \mathrm{~mJ} / \mathrm{cm}^{2}$ and a recording intensity of $5 \mathrm{~mW} / \mathrm{cm}^{2}$ at a spatial frequency of $1000 \mathrm{~L} / \mathrm{mm}$.

Two observations can be made from Table 3. Firstly, the final value of relative shrinkage is greater for the AA photopolymer than for the DA photopolymer for the same exposure energy. Secondly, $\eta$ growth is slightly faster in the AA photopolymer. This is explained by the relatively faster diffusion of the AA monomer molecules in comparison to the larger DA monomer molecules which will diffuse at a slower rate.

4.2. Results for Composition B: Effect of Zeolite Nanoparticles on Shrinkage. Measurement of the polymerisation-induced relative shrinkage of the BEA-doped AA photopolymer was carried out for four different concentrations of zeolite nanoparticles: $0,1,2.5$, and 5\% wt. All holographic recording was carried out using a total recording intensity of $5 \mathrm{~mW} / \mathrm{cm}^{2}$.

Figure 9 shows the relative shrinkage versus exposure time data for photopolymer layers doped with the different concentrations of BEA zeolites. From the data, it is evident that there is a decrease in relative shrinkage in the nanoparticle-doped photopolymer films.

As in Section 4.1, the experimental data was once again fitted with a double exponential function in order to quantify the different processes occurring in the photopolymer films during holographic recording. The values obtained from the fitted curve for the final relative shrinkage and the two time constants associated with the relative shrinkage are shown in Table 4. As for the DA photopolymer, the longer time constant $t_{2}$ is regarded as characteristic of the rate of monomer to polymer conversion.

The data in Table 4 shows that the amount of polymerisation-induced relative shrinkage occurring during 
TABLE 3: $\eta$ and relative shrinkage for the AA and DA photopolymers.

\begin{tabular}{lcccc}
\hline Exposure energy $\left(\mathrm{m} / \mathrm{cm}^{2}\right)$ & \multicolumn{2}{c}{ AA } & \multicolumn{2}{c}{ DA } \\
Relative shrinkage (\%)
\end{tabular}

TABLE 4: Data from double exponential fit of relative shrinkage versus exposure time for BEA-doped AA photopolymer.

\begin{tabular}{lcccrr}
\hline Nanoparticle concentration (\% wt.) & $y_{o}(\%)$ & $A_{1}$ & $t_{1}(\mathrm{~s})$ & $A_{2}$ & $t_{2}(\mathrm{~s})$ \\
\hline 0 & $3.40 \pm 0.05$ & $1.2 \pm 0.1$ & $9.50 \pm 0.02$ & $2.4 \pm 0.1$ & $62.50 \pm 0.02$ \\
1 & $3.15 \pm 0.04$ & $1.2 \pm 0.3$ & $2.70 \pm 0.09$ & $2.69 \pm 0.04$ & $55.50 \pm 0.01$ \\
2.5 & $2.94 \pm 0.04$ & $1.4 \pm 0.5$ & $2.2 \pm 0.1$ & $2.50 \pm 0.04$ & $50.00 \pm 0.01$ \\
5 & $3.34 \pm 0.05$ & $0.9 \pm 0.2$ & $4.30 \pm 0.08$ & $2.70 \pm 0.07$ & $58.80 \pm 0.01$ \\
\hline
\end{tabular}

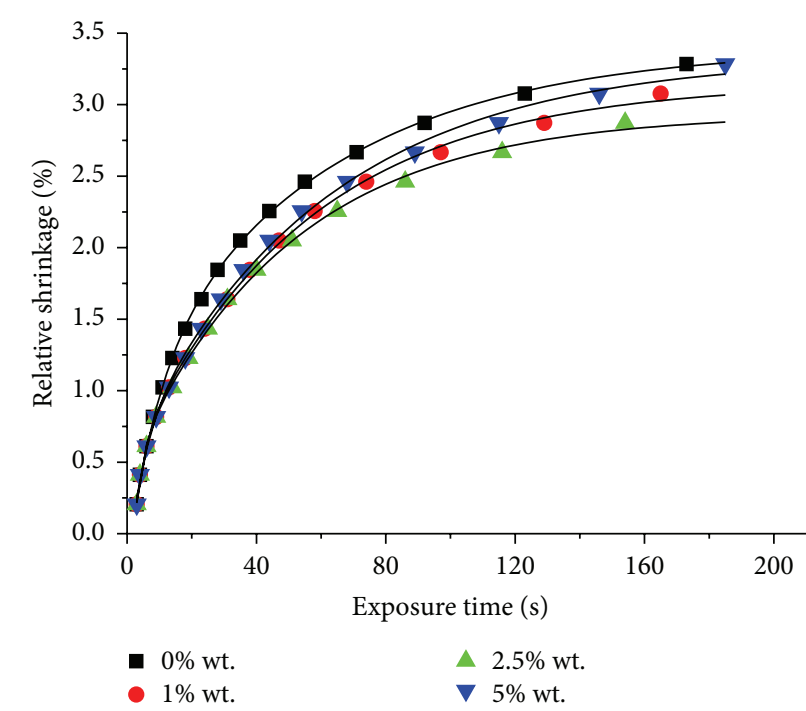

FIGURE 9: Relative shrinkage (\%) versus exposure time (s) for gratings recorded in $160 \mu \mathrm{m}$ thick AA photopolymer layers with BEA concentrations of $0,1,2.5$, and $5 \%$ wt.

holographic recording is reduced for all concentrations of BEA-zeolite nanoparticles in comparison to the undoped AA photopolymer layers. The lowest relative shrinkage value of $2.94 \%$ is achieved at a zeolite concentration of $2.5 \% \mathrm{wt}$. This represents a $13 \%$ decrease in relative shrinkage in comparison to the undoped photopolymer.

The observed reduction in relative shrinkage due to the incorporation of zeolites in the AA photopolymer may be attributed to increased stability of the nanoparticle-doped holographic grating. The redistribution of zeolite nanoparticles has previously been observed experimentally $[45,46]$. A periodic structure of zeolites throughout a photopolymer grating may act to increase the stability of the layer, thereby contributing to a reduction in polymerisationinduced shrinkage in the layer. The stability of the grating structure is enhanced as the zeolite concentration is increased up to $2.5 \%$ wt., resulting in a decrease in relative shrinkage.
As the concentration of zeolites is increased beyond the optimum value of $2.5 \% \mathrm{wt}$., the relative shrinkage is once again observed to increase. This may be due to increased scattering occurring in the layers as a result of an excessive volume of zeolite nanoparticles. Thus, the illumination pattern becomes similar to a single beam, leading to homogeneous polymerisation of layers and, therefore, greater relative shrinkage.

\section{Conclusions}

Modifications of the photopolymer composition in order to reduce polymerisation-induced shrinkage during holographic recording have been investigated. The first compositional change involving the replacement of the AA monomer with a larger monomer DA resulted in an average reduction in relative shrinkage of $10-15 \%$ in comparison to the AAbased photopolymer. This reduction is most likely due to the difference in the monomer molecule sizes and their respective diffusion rates, both of which govern the ability of the photopolymer media to produce long polymer chains. Both the absolute shrinkage and relative shrinkage in the DA photopolymer were observed to increase with increasing sample thickness. Higher recording intensities of 5 and $10 \mathrm{~mW} / \mathrm{cm}^{2}$ were observed to be favourable for minimising relative shrinkage of the DA photopolymer layers during holographic recording.

The second method tested for shrinkage reduction involved the incorporation of BEA-type zeolite nanoparticles in an AA-based photopolymer. A 13\% reduction in relative shrinkage was observed for photopolymer layers doped with an optimum concentration of $2.5 \%$ wt. of zeolite nanoparticles in comparison to the undoped AA photopolymer. The observed reduction in relative shrinkage is attributed to increased stability of the zeolite-doped grating structure.

Future work will include investigation of the effect of the incorporation of zeolite nanoparticles on the dynamics of the polymerisation-induced shrinkage for other photopolymer formulations, including the DA-based photopolymer. The 
effect of other nanoparticle types (e.g., $\mathrm{Au}$ and $\mathrm{Ag}$ ) on the relative shrinkage of the AA-based photopolymer will also be studied.

\section{Competing Interests}

The authors declare that there are no competing interests regarding the publication of this paper.

\section{Acknowledgments}

The authors acknowledge the Irish Research Council Embark Programme, the Enterprise Ireland-funded Project CF/2015/ 0076P, and Dublin Institute of Technology's Arnold F. Graves Postdoctoral Scholarship Scheme for funding this research.

\section{References}

[1] M. K. Kim, Digital Holographic Microscopy, Springer Series in Optical Sciences, 2011.

[2] F. Saglimbeni, S. Bianchi, A. Lepore, and R. Di Leonardo, "Three-axis digital holographic microscopy for high speed volumetric imaging," Optics Express, vol. 22, no. 11, pp. 13710-13718, 2014.

[3] M. Reicherter, S. Zwick, T. Haist, C. Kohler, H. Tiziani, and W. Osten, "Fast digital hologram generation and adaptive force measurement in liquid-crystal-display-based holographic tweezers," Applied Optics, vol. 45, no. 5, pp. 888-896, 2006.

[4] I. Naydenova, H. Akbari, C. Dalton et al., "Photopolymer holographic optical elements for application in solar energy concentrators," in Holography-Basic Principles and Contemporary Applications, E. Mihaylova, Ed., InTech, Rijeka, Croatia, 2013.

[5] A. Khan, N. R. Chakraborty, and H. L. Yadav, "Compound holographic optical element system for splitting and concentrating solar spectrum on laterally- arranged multiple band gap solar cells," International Journal of Physics and Applications, vol. 5, no. 3, pp. 115-120, 2013.

[6] A. K. Yetisen, I. Naydenova, F. Da Cruz Vasconcellos, J. Blyth, and C. R. Lowe, "Holographic sensors: three-dimensional analyte-sensitive nanostructures and their applications," Chemical Reviews, vol. 114, no. 20, pp. 10654-10696, 2014.

[7] I. Naydenova, R. Jallapuram, V. Toal, and S. Martin, "Characterisation of the humidity and temperature responses of a reflection hologram recorded in acrylamide-based photopolymer," Sensors and Actuators B: Chemical, vol. 139, no. 1, pp. 35-38, 2009.

[8] E. Leite, I. Naydenova, S. Mintova, L. Leclercq, and V. Toal, "Photopolymerizable nanocomposites for holographic recording and sensor application," Applied Optics, vol. 49, no. 19, pp. 3652-3660, 2010.

[9] J. Shi, V. K. S. Hsiao, and T. J. Huang, "Nanoporous polymeric transmission gratings for high-speed humidity sensing," Nanotechnology, vol. 18, no. 46, Article ID 465501, 2007.

[10] J. Blyth, R. B. Millington, A. G. Mayes, E. R. Frears, and C. R. Lowe, "Holographic sensor for water in solvents," Analytical Chemistry, vol. 68, no. 7, pp. 1089-1094, 1996.

[11] J. Guo, M. R. Gleeson, and J. T. Sheridan, "A review of the optimisation of photopolymer materials for holographic data storage," Physics Research International, vol. 2012, Article ID 803439, 16 pages, 2012.
[12] F.-K. Bruder, R. Hagen, T. Rölle, M.-S. Weiser, and T. Fäcke, "From the surface to volume: concepts for the next generation of optical-holographic data-storage materials," Angewandte Chemie-International Edition, vol. 50, no. 20, pp. 4552-4573, 2011.

[13] L. Shou-Jun, L. Guo-Dong, H. Qing-Sheng, and W. Min-Xian, "Holographic grating formation in dry photopolymer film with shrinkage," Chinese Physics, vol. 13, no. 9, pp. 1428-1431, 2004.

[14] J. E. Boyd, T. J. Trentler, R. K. Wahi, Y. I. Vega-Cantu, and V. L. Colvin, "Effect of film thickness on the performance of photopolymers as holographic recording materials," Applied Optics, vol. 39, no. 14, pp. 2353-2358, 2000.

[15] M. Atai and D. C. Watts, "A new kinetic model for the photopolymerization shrinkage-strain of dental composites and resin-monomers," Dental Materials, vol. 22, no. 8, pp. 785-791, 2006.

[16] S. Lee, Y.-C. Jeong, Y. Heo, S. I. Kim, Y.-S. Choi, and J.-K. Park, "Holographic photopolymers of organic/inorganic hybrid interpenetrating networks for reduced volume shrinkage," Journal of Materials Chemistry, vol. 19, no. 8, pp. 1105-1114, 2009.

[17] J. T. Gallo and C. M. Verber, "Model for the effects of material shrinkage on volume holograms," Applied Optics, vol. 33, no. 29, pp. 6797-6806, 1994.

[18] H. J. Coufal, D. Psaltis, and G. T. Sincerbox, Holographic Data Storage, Springer Series in Optical Sciences, Springer, Berlin, Germany, 2000.

[19] M. Moothanchery, I. Naydenova, and V. Toal, "Studies of shrinkage as a result of holographic recording in acrylamidebased photopolymer film," Applied Physics A, vol. 104, no. 3, pp. 899-902, 2011.

[20] D. Cody, I. Naydenova, and E. Mihaylova, "New non-toxic holographic photopolymer material," Journal of Optics, vol. 14, no. 1, article 015601, 2012.

[21] D. Cody, I. Naydenova, and E. Mihaylova, "Effect of glycerol on a diacetone acrylamide-based holographic photopolymer material," Applied Optics, vol. 52, no. 3, pp. 489-494, 2013.

[22] D. Cody, E. Mihaylova, L. O’Neill, and I. Naydenova, "Determination of the polymerisation rate of a low-toxicity diacetone acrylamide-based holographic photopolymer using Raman spectroscopy," Optical Materials, vol. 48, pp. 12-17, 2015.

[23] M. Moothanchery, Studies of shrinkage in photopolymerisable materials for holographic applications [Ph.D. thesis], Dublin Institute of Technology, Dublin, Ireland, 2013.

[24] H. Krug and H. Schmidt, "Organic-inorganic nanocomposites for micro optical applications," New Journal of Chemistry, vol. 18, pp. 1125-1134, 1994.

[25] P. W. Oliveira, H. Krug, P. Müller, and H. Schmidt, "Fabrication of GRIN-materials by photopolymerization of diffusioncontrolled organic-inorganic nanocomposite materials," MRS Proceedings, vol. 435, p. 553, 1996.

[26] N. Suzuki, Y. Tomita, and T. Kojima, "Holographic recording in $\mathrm{TiO}_{2}$ nanoparticle-dispersed methacrylate photopolymer films," Applied Physics Letters, vol. 81, no. 22, pp. 4121-4123, 2002.

[27] N. Suzuki and Y. Tomita, "Silica-nanoparticle-dispersed methacrylate photopolymers with net diffraction efficiency near 100\%," Applied Optics, vol. 43, no. 10, pp. 2125-2129, 2004.

[28] N. Suzuki, Y. Tomita, K. Ohmori, M. Hidaka, and K. Chikama, "Highly transparent $\mathrm{ZrO}_{2}$ nanoparticle-dispersed acrylate photopolymers for volume holographic recording," Optics Express, vol. 14, no. 26, pp. 12712-12719, 2006. 
[29] C. Sánchez, M. J. Escuti, C. Van Heesch et al., “ $\mathrm{TiO}_{2}$ nanoparticle-photopolymer composites for volume holographic recording," Advanced Functional Materials, vol. 15, no. 10, pp. 1623-1629, 2005.

[30] Y. Tomita, E. Hata, K. Momose et al., "Photopolymerizable nanocomposite photonic materials and their holographic applications in light and neutron optics," Journal of Modern Optics, vol. 63, supplement 3, pp. S1-S31, 2016.

[31] T. J. Trentler, J. E. Boyd, and V. L. Colvin, "Epoxy resinphotopolymer composites for volume holography," Chemistry of Materials, vol. 12, no. 5, pp. 1431-1438, 2000.

[32] E. Hata and Y. Tomita, "Order-of-magnitude polymerizationshrinkage suppression of volume gratings recorded in nanoparticle-polymer composites," Optics Letters, vol. 35, no. 3, pp. 396-398, 2010.

[33] M. Moothanchery, I. Naydenova, S. Mintova, and V. Toal, "Nanozeolites doped photopolymer layers with reduced shrinkage," Optics Express, vol. 19, no. 25, pp. 25786-25791, 2011.

[34] C. Zhao, J. Liu, Z. Fu, and R. T. Chen, "Shrinkage correction of volume phase holograms for optical interconnects," in Optoelectronic Interconnects and Packaging IV, vol. 3005 of Proceedings of SPIE, pp. 224-229, San Jose, Calif, USA, February 1997.

[35] R. M. Shelby, D. A. Waldman, and R. T. Ingwall, "Characterization of a low-shrinkage photopolymer for holographic data storage," in Proceedings of the Conference on Lasers and ElectroOptics (CLEO '00), San Francisco, Calif, USA, May 2000.

[36] S. Gallego, A. Márquez, D. Méndez et al., "Analysis of PVA/AA based photopolymers at the zero spatial frequency limit using interferometric methods," Applied Optics, vol. 47, no. 14, pp. 2557-2563, 2008.

[37] M. Kawana, J. Takahashi, J. Guo, and Y. Tomita, "Measurement of polymerization-shrinkage evolution during curing in photopolymer with a white-light Fabry-Pérot interferometer," Optics Express, vol. 23, no. 12, pp. 15356-15364, 2015.

[38] E. N. Leith and J. Upatnieks, "Wavefront reconstruction with continuous-tone objects," Journal of the Optical Society of America, vol. 53, no. 12, pp. 1377-1381, 1963.

[39] R. L. Powell and K. A. Stetson, "Interferometric vibration analysis by wavefront reconstruction," Journal of the Optical Society of America, vol. 55, pp. 1593-1598, 1965.

[40] R. K. Erf, Holographic Non-Destructive Testing, Academic Press, London, UK, 1974.

[41] R. Jones and C. Wykes, Holographic and Speckle Interferometry, Cambridge University Press, Cambridge, UK, 1983.

[42] P. K. Rastogi, Optical Measurement Technique and Applications, Artech House, Norwood, Mass, USA, 1997.

[43] R. C. Sampson, "Holographic-interferometry applications in experimental mechanics," Experimental Mechanics, vol. 10, no. 8, pp. 313-320, 1970.

[44] M. Moothanchery, V. Bavigadda, V. Toal, and I. Naydenova, "Shrinkage during holographic recording in photopolymer films determined by holographic interferometry," Applied Optics, vol. 52, no. 35, pp. 8519-8527, 2013.

[45] I. Naydenova, E. Leite, T. Z. Babeva et al., "Optical properties of photopolymerizable nanocomposites containing nanosized molecular sieves," Journal of Optics, vol. 13, no. 4, Article ID 044019, 2011.

[46] A. M. Ostrowski, I. Naydenova, and V. Toal, "Light-induced redistribution of Si-MFI zeolite nanoparticles in acrylamidebased photopolymer holographic gratings," Journal of Optics A: Pure and Applied Optics, vol. 11, no. 3, Article ID 034004, 2009. 

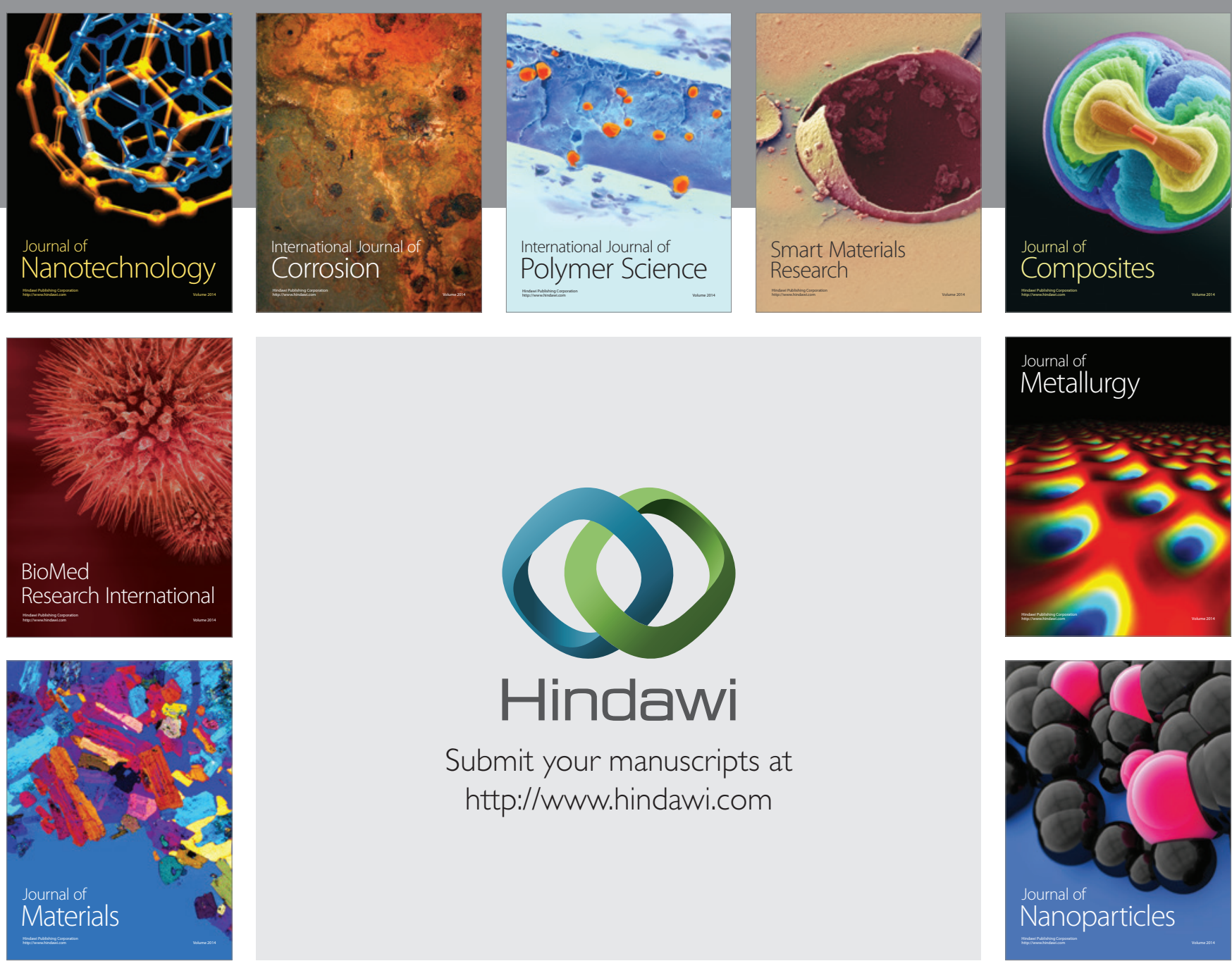

\section{Hindawi}

Submit your manuscripts at

http://www.hindawi.com

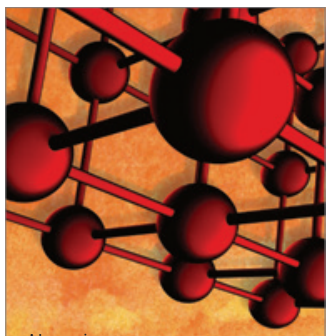

Materials Science and Engineering
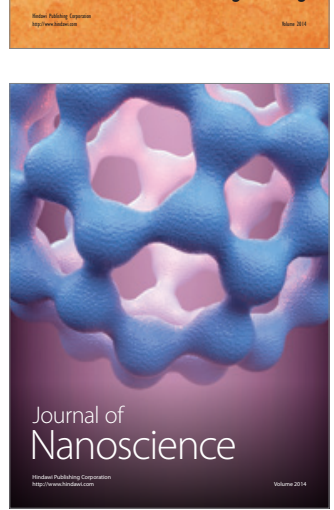
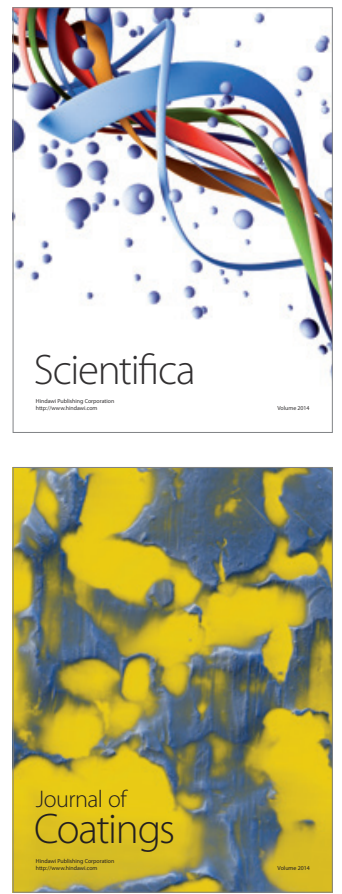
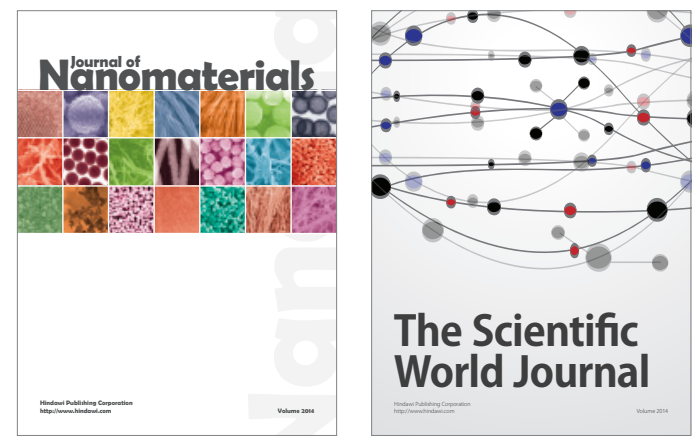

The Scientific World Journal
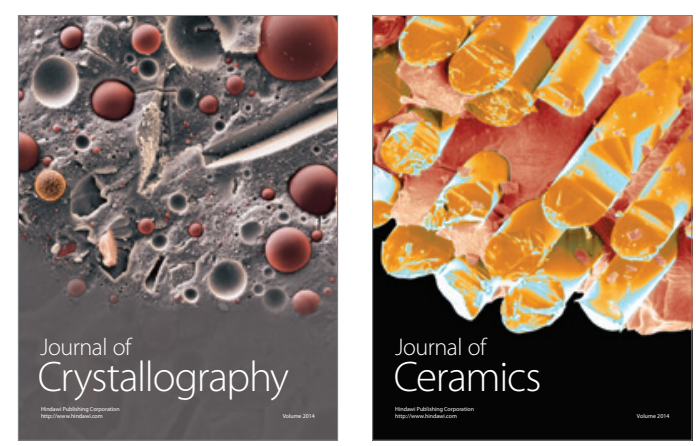
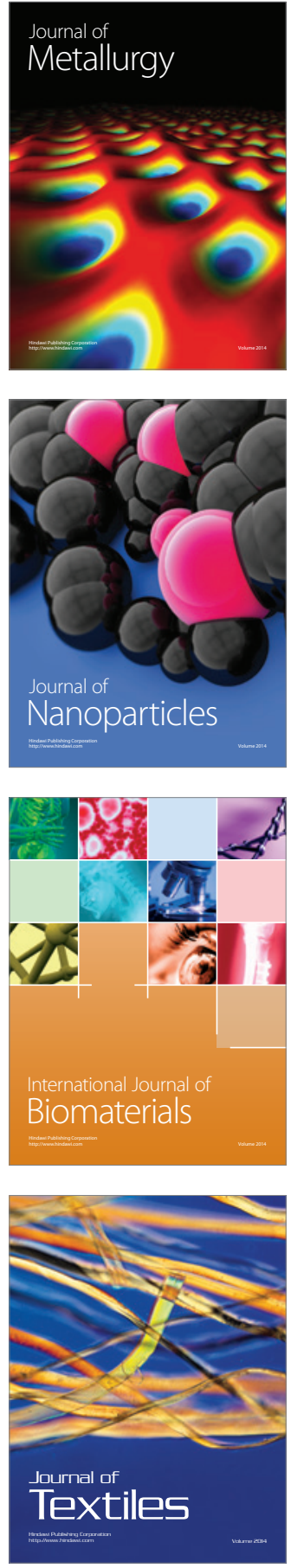